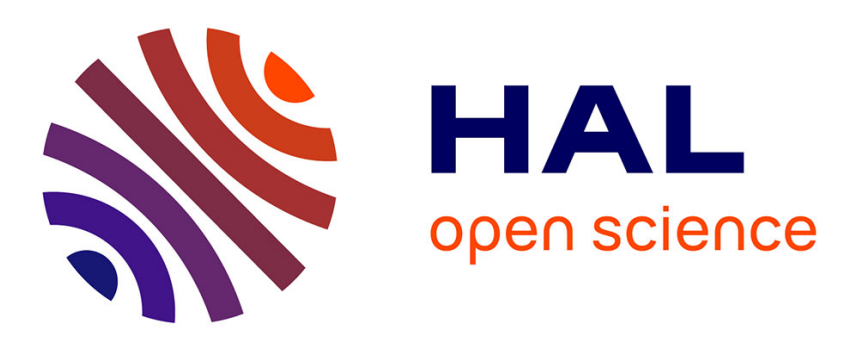

\title{
Radiation-induced micro-structures as ground states of a Swift-Hohenberg energy functional
}

D. Simeone, G. Thorogood, G. L. Murphy, A. Forestier, P. Garcia, L. Luneville

\section{To cite this version:}

D. Simeone, G. Thorogood, G. L. Murphy, A. Forestier, P. Garcia, et al.. Radiation-induced microstructures as ground states of a Swift-Hohenberg energy functional. Journal of Applied Physics, 2019, 125 (6), pp.065103. 10.1063/1.5072798 . cea-02510015

\section{HAL Id: cea-02510015 https://hal-cea.archives-ouvertes.fr/cea-02510015}

Submitted on 17 Mar 2020

HAL is a multi-disciplinary open access archive for the deposit and dissemination of scientific research documents, whether they are published or not. The documents may come from teaching and research institutions in France or abroad, or from public or private research centers.
L'archive ouverte pluridisciplinaire HAL, est destinée au dépôt et à la diffusion de documents scientifiques de niveau recherche, publiés ou non, émanant des établissements d'enseignement et de recherche français ou étrangers, des laboratoires publics ou privés. 


\title{
Radiation-induced micro-structures as ground states of a Swift-Hohenberg energy functional
}

\author{
D. Simeone, ${ }^{1}$ G.J. Thorogood ${ }^{2}$ G.L. Murphy, ${ }^{3}$ A. Forestier, ${ }^{4}$ P. Garcia, ${ }^{5}$ and L. Luneville ${ }^{6}$ \\ 1) CEA/DEN/DMN/SRMA/LA2M-LRC CARMEN, CEA, Université Paris-Saclay, \\ F-91191, Gif-sur-Yvette, France, CNRS/ECP/UMR 8085, Grande voie des vignes, \\ Chatenay Malabry France \\ ${ }^{2)}$ ANSTO, Lucas Heights, NSW, Australia and Department of Nuclear System Safety Engineering, \\ Nagaoka University of Technology, 1603-1 Kamitomioka, Nagaoka 940-2188, \\ Japan \\ 3) ANSTO, Lucas Heights, NSW, Australia and Department of Nuclear System Safety Engineering, \\ School of Chemistry, The University of Sydney, Sydney, NSW 2006, Australia \\ ${ }^{4)}$ CEA/DEN/DANS, Université Paris-Saclay, F-91191, Gif-sur-Yvette \\ ${ }^{5)}$ CEA, DEN, DEC, Centre de Cadarache, 13108, Saint-Paul-Lez-Durance Cedex \\ 6) CEA/DEN/DM2S/SERMA/LLPR-LRC CARMEN, CEA, Université Paris-Saclay, \\ F-91191, Gif-sur-Yvette, France,CNRS/ECP/UMR 8085, Grande voie des vignes, \\ Chatenay Malabry France France
}

(Dated: 18 October 2018)

We demonstrate that the Swift-Hohenberg functional as used to describe patterning observed in out of equilibrium systems such as diblock copolymers, Rayleigh-Benard convection and thin film magnetic garnets, can be applied to radiation-induced patterns that occur in non-miscible alloys. By comparing ground states obtained from the minimization of this functional and a $2 \mathrm{D}$ numerical simulation performed on an irradiated $\mathrm{AgCu}$ material which is the archetype of a non-miscible alloy we show that the Swift-Hohenberg functional provides all possible patterns generated under irradiation and the solubility limits of radiation-induced precipitates in these patterns. To rationalize the formation of these radiation-induced patterns, we propose a generic "pseudo phase diagram" that relies not only on the irradiation flux and temperature but also the overall composition of the alloy. Tuning this overall composition offers the opportunity to tailor new materials with various micro-structures overcoming the limitation of the equilibrium phase diagram.

Materials ranging from ferro-fluids to polymers, when at equilibrium, can exhibit distinct patterns ${ }^{1,2}$ which can be computed from ground states of their free en$\mathrm{ergy}^{2,3}$. In systems far from equilibrium, i.e. under irradiation, experimental observations have also revealed the existence of such patterns ${ }^{4,5}$. Experimental irradiationinduced dislocation lattices ${ }^{6}$ can be readily modelled via reaction-diffusion type equations within a chemical rate theory framework ${ }^{6,7}$. However, there is presently no overarching theory that predicts and models the patterns resulting from irradiation-induced disordering.

At the atomic scale, Molecular dynamics (MD) is used to predict how metals and oxides will behave when exposed to radiation over inter atomic distances; its strength lies in the ability to predict the initial stage of radiation damage. It is however limited to pico-second ranges and small simulation boxes $\left(10^{6}\right.$ atoms). Further along the length and time scale lies Kinetic Monte-Carlo $(\mathrm{KMC})^{8,9}$, it is capable of describing point defects and dislocations. However, this method is still limited by the size of the simulation boxe and time ${ }^{7}$. Due to these limitations, attempts have been made to develop modelling of radiation-induced disordering at larger space and time scales within the Phase Field framework ${ }^{7,10-12}$.

In this study we have examined the formation of radiation-induced disordering patterns within the Phase Field framework, the continuum counterpart of the atomic Ising model extensively used in KMC simulations of radiation-induced disordering ${ }^{8,13}$. The postulate, com- monly admitted in the radiation damage community, is that radiation-induced patterns can emerge from the balance of disordering effects induced by irradiation (hereafter called ballistic effects ${ }^{7}$ controlled by the irradiation flux $\phi$ ) and radiation enhanced diffusion processes tuned by the temperature $T$. This balance leads to the formation of unexpected patterns when compared with those predicted under the thermodynamic equilibrium ${ }^{10,14}$.

This work highlights that the Swift-Hohenberg (SH) functional ${ }^{15}$, extensively used to discuss the formation and the stability of patterns in materials produced out of equilibrium ${ }^{2,3}$, can predict the appearance of all possible ground states (microstructures) associated with these radiation-induced patterns. The novelty of this study lies in two aspects. First, we illustrate with an example $(\mathrm{AgCu}$ non miscible alloy) that with our analytic approach to the $\mathrm{SH}$ functional it is possible to predict the entire set of patterns that may be produced under irradiation $^{4,16}$. Secondly, we generalize the "pseudo phase diagram" generated under irradiation to include, along with temperature and incident particle flux, the overall composition of the alloy, the effect of which has been overlooked in previous studies ${ }^{10,14}$.

Disordering of non-miscible $A_{\bar{c}} B_{1-\bar{c}}$ alloys of overall composition $\bar{c}$ via irradiation is the result of a redistribution of atomic species via their thermal mobility enhanced by vacancies produced under irradiation $\Gamma_{t h}(T, \phi)^{12}$ and atom relocation which is triggered by ballistic effects due to atomic collisions in the crystal, 
$\Gamma_{\text {irr }}(\phi)^{10,14}$. At the mesoscale, the local composition $c(\mathbf{r}, t)$ of species $\mathrm{A}$ in the alloy, is described by the scalar order parameter field $\eta(\mathbf{r}, t)=c(\mathbf{r}, t)-c_{0}$ where $c_{0}$ is the atomic fraction of species $\mathrm{A}$ at the critical temperature $T_{0}$, i.e. the temperature for which the order parameter is null. For $\mathrm{AgCu}$ alloys, $c_{0}$ is equal to 0.38 and the critical temperature fitted the experimental equilibrium phase diagram ${ }^{11}$ is equal to $1200 K^{17}$.

At low temperatures, $\left(T<<T_{0}\right)$, the thermal evolution of the alloy follows a spinodal decomposition as given by $^{18}$ :

$$
\left.\frac{\partial \eta(\mathbf{r}, t)}{\partial t}\right|_{t h}=\Gamma_{t h}(T, \phi) \nabla^{2} \frac{\delta F[\eta]}{\delta \eta}
$$

where $F[\eta]=\int f(\eta(\mathbf{r}, t)) d \mathbf{r}$ represents the free energy of the system. In this expression, the free energy density, $f(\eta(\mathbf{r}, t))$, is represented by a Landau fourth order expansion, $\frac{a_{2}}{2} \eta(\mathbf{r}, t)^{2}+\frac{a_{3}}{3} \eta(\mathbf{r}, t)^{3}+\frac{a_{4}}{4} \eta(\mathbf{r}, t)^{4}$. The third order term is negative as expected for a decomposition transformation, which is a first order transition ${ }^{18,19}$.

The spatial heterogeneity of $\eta(\mathbf{r}, t)$ is represented by adding to $F[\eta]$ the Ginzburg term, $\int \kappa|\nabla \eta(\mathbf{r}, t)|^{2} d \mathbf{r}$, where $\kappa$, is the energetic cost of interfaces forming between separate domains of different compositions ${ }^{18}(\kappa>$ $0)$.

Alternatively, the dynamics of $\eta(\mathbf{r}, t)$ induced by the ballistic effects is non local and can be modelled via ${ }^{20,21}$ :

$$
\left.\frac{\partial \eta(\mathbf{r}, t)}{\partial t}\right|_{i r r}=\Gamma_{i r r}(\phi)\left[\int p_{R_{p}}\left(\mathbf{r}-\mathbf{r}^{\prime}\right) \eta\left(\mathbf{r}^{\prime}, t\right) d r^{\prime}-\eta(\mathbf{r}, t)\right]
$$

where, $p_{R_{p}}(\mathbf{r})$, is the probability density of an atom relocating in a displacement cascade which lasts a few hundreds of pico-seconds and $R_{p}$ is the mean free path of the relocated atom ${ }^{10,14}$. $R_{p}$ depends on the energy, the nature of the incident projectiles and the atomic composition of the target. For instance, $R_{p}$ is equal to 0.3 nanometers for $\mathrm{AgCu}$ samples irradiated by $1 \mathrm{MeV} \mathrm{Kr}$ ions.

Eq.( 1) and Eq.( 2) result from a space and time coarsegraining method ${ }^{10,21}$. In the Phase Field framework, the coarse-grained time scale is controlled by the diffusion of atoms (few $10^{-6}$ seconds, i.e. 4 orders of magnitude larger than the expansion of a displacement cascade) and the space scale is proportional to $\kappa^{-1}$ which is one order of magnitude larger than $R_{p}$. The main interest of this approach is to incorporate long range elastic fields induced by precipitates ${ }^{18}$.

The specific form of Eq.( 2) enables the modelling of two dynamics, acting in parallel as expected from the postulate, via a relaxation equation ${ }^{12,14}$ :

$$
\left.\frac{\partial \eta(\mathbf{r}, t)}{\partial t}\right|_{t o t}=\Gamma_{t h}(T, \phi) \nabla^{2} \frac{\partial \mathcal{L}[\eta]}{\partial \eta}
$$

where $\mathcal{L}[\eta]=F[\eta]+\frac{\Gamma_{i r r}(\phi)}{2 \Gamma_{t h}(T, \phi)} G[\eta]$ can be understood as the Lyapounov functional for the full set of dynamics. In this functional, the effect of non-local ballistic effects is described by $G(\eta(\mathbf{r}, t))=\iint \eta(\mathbf{r}, t) g\left(\mathbf{r}-\mathbf{r}^{\prime}\right) \eta\left(\mathbf{r}^{\prime}, t\right) d \mathbf{r} d \mathbf{r}^{\prime}$ (where $\nabla^{2} g=\left(p_{R_{p}}-\delta\right)$ ). The dynamics behaves as if it was at $T=0$ with a temperature dependence only entering through T-dependent model parameters and is only subject to random initial conditions associated with a fully disordered $\operatorname{state}^{22}$. The challenge of understanding radiation-induced disordering therefore can be posed as finding the nature of ground states of $\mathcal{L}[\eta]$ under the constraint of conservation of the overall value, $\bar{\eta}$, of the order parameter $\eta$.

Introducing a space characteristic length, $l_{0}=\sqrt{\frac{\left|a_{2}\right|}{a_{4} \alpha^{2}}}$ (with $\alpha=\frac{\eta_{+}-\eta_{-}}{2}\left(\eta_{ \pm}\right.$are the solubility limits of the equilibrium phase diagram), a dimensionless Lyapounov functional $\mathcal{L}^{\prime}[\eta]=\frac{\mathcal{L}[\eta]}{F_{0}}\left(F_{0}=\frac{\alpha \kappa^{\frac{3}{2}}}{\sqrt{a_{4}}}\right)$ can be computed as a function of the reduced scalar order parameter field $\eta(\mathbf{r}, t)=\frac{c(\mathbf{r}, t)-c_{0}}{\alpha}$, the enhancement factor $\Delta(\phi, T)=$ $\frac{\Gamma_{i r r}(\phi)}{\Gamma_{t h}(\phi, T)} \frac{\kappa}{\alpha^{4} a_{4}{ }^{2} \Omega_{a t}}\left(\Omega_{a t}\right.$ is the mean atomic volume of the alloy) and the dimensionless mean free path $R=\frac{R_{p}}{l_{0}}$. A universal "pseudo phase diagram" can be derived from the minimization of the quadratic part of $\mathcal{L}^{\prime}[\eta]=$ $\int \frac{D(k)}{2}|\hat{\eta}(\mathbf{k}, t)|^{2} d \mathbf{k}$ (where $D(k)=-1+k^{2}+\frac{\Delta R^{2}}{1+R^{2} k^{2}}$ and $\hat{\eta}(\mathbf{k}, t)$ is the Fourier transform of $\eta(\mathbf{x}, t))$. This univer-

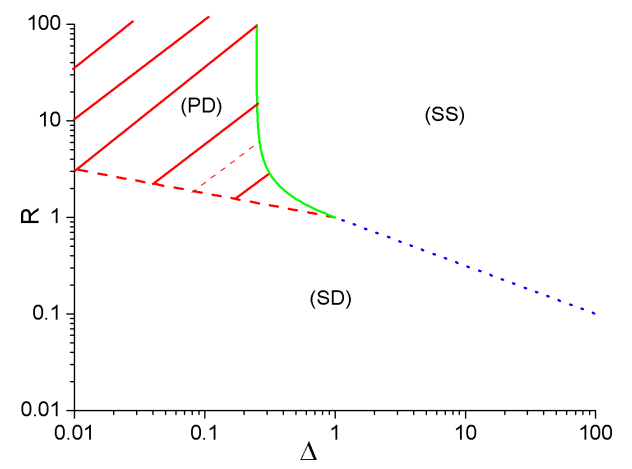

FIG. 1. "Pseudo phase diagram" resulting from the irradiation of non-miscible alloys as a function of the reduced units $\Delta$ and $R$ irrespective of their overall composition. Three distinct domains can be produced : a disordered domain associated with a solid solution (SS), a domain where the spinodal decomposition (SD) takes place and a pattern domain (PD) where the coarsening of precipitates is frozen. (dashed red line: limit between the spinodal decomposition and patterning of domains $\left(\Delta_{m i n}\right)$, blue dotted line: limit between the $(\mathrm{PD})$ and the $(\mathrm{SS}))$

sal "pseudo phase diagram", spanned by $R$ and $\Delta$, exhibits three distinct domains associated with two "limit of phases" $\Delta_{\min }=R^{-4}$ (dashed line in Fig 1) and $\Delta_{\max }=\frac{\left(1+R^{2}\right)^{2}}{4 R^{4}}$ (full line in Fig 1$)$. At high $R$ values, ballistic effects dominate chemical effects and disordering of the alloy occurs leading to a solid solution (SS). At low $R$ values, the ballistic effects are neglected in comparison with the chemical nature of species forming the alloy and a spinodal decomposition (SD) occurs. In this do- 
main, A-rich precipitates of characteristic size $L(t) \propto t^{\frac{1}{3}}$ coarsen $^{23}$. At low temperatures and large relocation distances (PD area), i.e. for $\Delta_{\min }<\Delta<\Delta_{\max }$, A-rich precipitates stop to coarsen and exhibit a characteristic size $\frac{2 \pi}{k_{0}}$. This composition modulation is defined by a non null wave vector $\mathbf{k}_{\mathbf{0}}$ of modulus $k_{0}=\left(\sqrt{\Delta}-\sqrt{\Delta_{\min }}\right)^{\frac{1}{2}}$. The limit between the disordered (SS) and ordered phases (SD and $\mathrm{PD}) \Delta_{\max }$ is given by $D\left(k_{0}\right)=0$, in perfect agreement with the previous limit derived from a linear analysis of the growth rate of Eq. $(3)^{14}$. The limit between (SD) and (PD) domains $\Delta_{\text {min }}$, given by the vanishing of $k_{0}{ }^{10}$, differs from the previous one calculated assuming that $\eta(\mathbf{r})$ exhibits a tanh like shape ${ }^{14}$.

Even if this analysis is sufficient to construct a universal "pseudo phase diagram", information about the possible micro-structures (stripes, droplets...) that emerge in the (PD) area are not revealed. Additionally, as Eq. (3) is a conservative equation, it is conceivable that the overall order parameter $\bar{\eta}$, is a selection factor that determines the nature of all possible radiation-induced patterns, this dependence does not appear in Fig 1.

To describe micro-structures in the (PD) area $\left(\Delta_{\min }<\right.$ $\left.\Delta<\Delta_{\max }\right)$, a structure factor $S(k, t)=<|\hat{\eta}(\mathbf{k}, t)|^{2}>$ (where $<.>$ is a radial average) can be extracted from the numerical resolution of Eq.( 3) obtained by fixing the first and third spatial derivatives of $\eta(\mathbf{x}, t)$ to zero at the boundaries. An example of this structure factor is plotted in Fig. 2 for an $A g_{0.38} C u_{0.62}$ alloy $(\bar{\eta}=0)$ irradiated at $440 \mathrm{~K}$ by $1 \mathrm{MeV} \mathrm{Kr}$ ions (values of $a_{j}, \kappa, \Gamma_{i r r}(\phi)$ and $\Gamma_{t h}(T, \phi)$ used in our $2 \mathrm{D}$ simulations can be found in Ref. $\left.{ }^{11}\right)$. The 2D steady-state pattern shown in the left-hand side of Fig. 2 was obtained by fixing the overall composition $(\bar{\psi}=0)$ and is in fair agreement with $2 \mathrm{D}$ patterns predicted by $\mathrm{KMC}$ simulations for the $\mathrm{AgCu}$ al$\operatorname{loy}^{24}$ in the Bragg-William approximation, assessing our Phase Field approach. At late-times $\left(t \Gamma_{t h}(T, \phi)>>1\right)$, the structure factor $S(k, t) \propto D(k)^{-1}$, displays a peak around $k_{0}$ (white rings in Fig.2) and the quadratic part of $\mathcal{L}^{\prime}[\eta]$ reduces to $\int \frac{1}{2} \eta\left(\mathbf{r}_{2}\right)\left[D\left(k_{0}\right)+\left(1-\sqrt{\frac{\Delta_{\min }}{\Delta}}\right) k_{0}^{-2}\left(\nabla^{2}+\right.\right.$ $\left.\left.k_{0}^{2}\right)^{2}\right] \eta\left(\mathbf{r}_{\mathbf{1}}\right) d \mathbf{r}_{\mathbf{1}} d \mathbf{r}_{\mathbf{2}}$ which is no more than the quadratic part of the Swift-Hohenberg functional extensively used to model pattern formation in non-equilibrium systems ${ }^{3}$. Ground states of the SH functional are defined by periodic modulations of $\eta(\mathbf{r}, t)$ associated with a wave vector $\mathbf{k}_{\mathbf{0}}$ and differ from the homogeneous ground states derived from the minimization of the Cahn-Hilliard equation $^{23}$.

Introducing a new space length $\left(r_{i}^{\prime}=k_{0} r_{i}\right), \mathcal{L}^{\prime}[\eta]$ reduces to the standard $\mathrm{SH}$ form ${ }^{3,15,25}$ :

$$
\begin{aligned}
\mathcal{L}_{S H}[\psi] & =\int \frac{\psi\left(\mathbf{r}_{\mathbf{2}}^{\prime}\right)}{2}\left[-\epsilon(R, \Delta)+\left(1+\nabla^{2}\right)^{2}\right] \psi\left(\mathbf{r}_{\mathbf{1}}^{\prime}\right) d r_{1}^{\prime} d r_{2}^{\prime} \\
& +\int \frac{\psi\left(\mathbf{r}_{\mathbf{1}}^{\prime}\right)^{4}}{4} d r_{1}^{\prime}
\end{aligned}
$$

where $\psi$ is the reduced scalar order parameter field $\left(\psi=\left(\eta+\frac{a_{3}}{a_{4}}\right)\left(1-\sqrt{\frac{\Delta_{\min }}{\Delta}}\right)^{-1 / 2}\left(k_{0} \alpha\right)^{-1}\right)$ and $\mathcal{L}_{S H}$ is re-
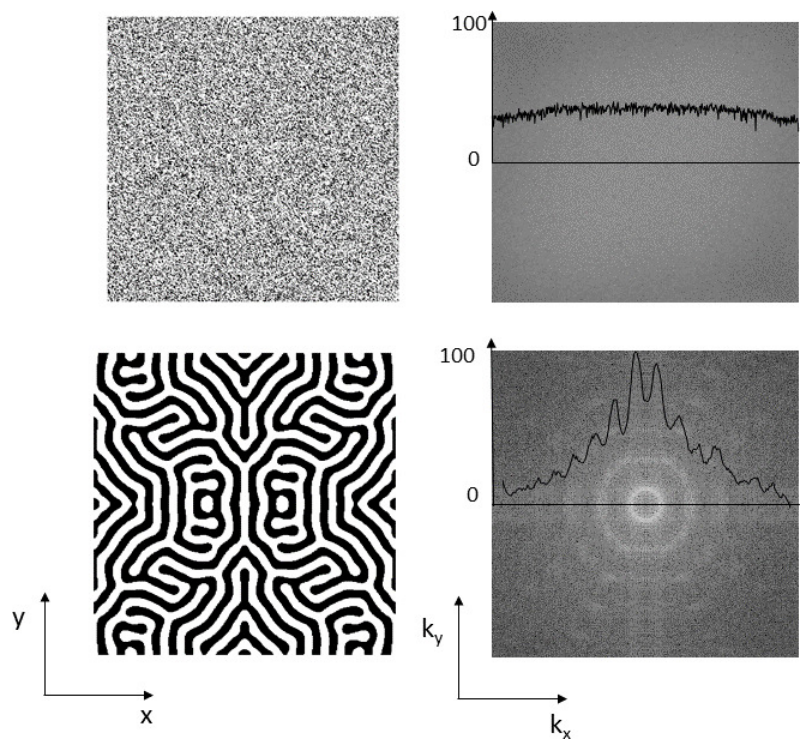

FIG. 2. 2D simulation of a random distribution of a $A g_{0.38} C u_{0.62}$ alloy, before (top ) and after (bottom ) irradiation with $1 \mathrm{MeV} \mathrm{Kr}$ ions at $\phi=6 \times 10^{12} \mathrm{~cm}^{-2} \mathrm{~s}^{-1}$, and $T=440 K$ obtained from the numerical solution of Eq.( 3 ) ( $R=3$ and $\Delta=0.2$ ). Initial (up left-hand side) and final (down left-hand side) 2D micro-structures in real space and their corresponding Power Spectral Density (PSD) in the Fourier space (right-hand side) are plotted. In these simulations, A rich (black) (B rich (white)) domains are associated with positive (negative) $\eta$ values (the $2 \mathrm{D}$ domain size is equal to 200 in reduced units). At late-time, the PSD is radially symmetric and peaks sharply around $k_{0}$ (intense white ring). Late-time structure factors $S(k, t)$, resulting from a circular average of the PSD for both initial (top) and final microstructures (bottom), are plotted as a full line along the $k_{x}$ component of the wave vector.

scaled by $\left(1-\sqrt{\frac{\Delta_{\text {min }}}{\Delta}}\right)^{-2} k_{0}$. In this expression, $\epsilon(R, \Delta)=$ $\frac{2 \sqrt{\Delta}\left(\sqrt{\Delta_{\max }}-\sqrt{\Delta}\right)}{\left(\sqrt{\Delta}-\sqrt{\Delta_{\text {min }}}\right)^{2}}$ contains all the information about the global dynamics. This approximation of the Lyapounov functional by a $\mathrm{SH}$ functional $\mathcal{L}_{S H}$ in the pattern domain is the keystone result of this study. Ground states of the SH functional are not only a function of $\epsilon(R, \delta)$ but also of the overall composition of the alloy $\bar{\psi}$ (in reduced units) which has been neglected in previous studies ${ }^{10,14}$. This implies that a more generic "pseudo phase diagram" including the overall composition of the alloy can be drawn (Fig.3) to extend previous studies.

$2 \mathrm{D}$ ground states ${ }^{26,27}$, resulting from the minimization of $\mathcal{L}_{S H}$, can be analytically computed:

- a uniform micro-structure with $\psi(\mathbf{r})=\bar{\psi}$ (graph 6 in Fig. 3)

- a labyrinthine micro-structure (stripes) with compositional fluctuations, $\psi(\mathbf{r})=\bar{\psi}+A_{s} \cos (\mathbf{k r})$ and wave vectors, $\mathbf{k}=k_{0}\left(\begin{array}{l}1 \\ 0\end{array}\right)$ and $\mathbf{k}=k_{0}\left(\begin{array}{l}0 \\ 1\end{array}\right)$ (graph 1 in Fig. 3). In the one mode approximation ${ }^{6}$, the 
amplitude of the modulation is equal to ${ }^{26} A_{s}=$ $\frac{2}{3} \sqrt{3 \epsilon(R, \Delta)-9 \bar{\psi}^{2}}$

- a honeycomb structure (hexagonal dots) with compositional fluctuations $\psi(\mathbf{r})=\bar{\psi}+A_{h} \sum_{j=1}^{3} e^{i \mathbf{k}_{\mathbf{j}} \cdot \mathbf{r}}+$ c.c. and wave vectors, $\mathbf{k}_{\mathbf{1}}=k_{0}\left(\begin{array}{l}1 \\ 0\end{array}\right), \mathbf{k}_{\mathbf{2}}=$ $k_{0}\left(\begin{array}{c}-1 / 2 \\ \sqrt{3} / 2\end{array}\right)$ and $\mathbf{k}_{\mathbf{3}}=k_{0}\left(\begin{array}{c}-1 / 2 \\ -\sqrt{3} / 2\end{array}\right)$ (graph 3 in Fig. 3). In the one mode approximation ${ }^{6}$, the amplitude of the modulation is equal to ${ }^{26} A_{h}=$ $\frac{4}{5}\left[\bar{\psi}+\frac{\sqrt{15 \epsilon(R, \Delta)-36 \bar{\psi}^{2}}}{3}\right]$

These 2D ground states are in very good agreement with snapshots of late-time micro-structures extracted from our 2D numerical simulations (graphs in Fig.3). Labyrinthine lamelar stripes forming at low $|\bar{\psi}|$ (snapshot
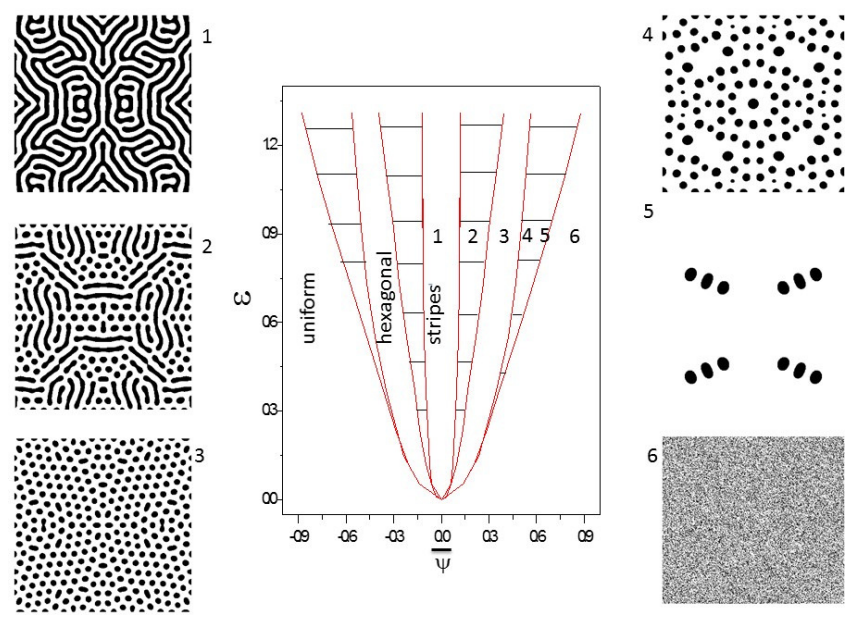

FIG. 3. Proposed generic 2D "Pseudo phase diagram" in the (PD) area derived from the SH functional as function of the nominal composition, $\bar{\psi}$ and the $\epsilon(R, \Delta)$ parameter. The solid lines represent the limits between different micro-structures and the hatched area is associated with coexistent domains. This "pseudo phase diagram" is in fair agreement with $2 \mathrm{D}$ simulated microstructures (graphs 1 to 6) resulting from the numerical resolution of Eq. (3) (From top-left to bottom-right, 1: $\bar{\psi}=0,2: \bar{\psi}=0.2,3: \bar{\psi}=0.35,4: \bar{\psi}=0.5,5: \bar{\psi}=0.65,5$ : $\bar{\psi}=0.7$ and $\epsilon=0.85$ ).

1 in Fig. 3) evolve to a honeycomb structure of spherical precipitates forming for intermediate $|\bar{\psi}|$ values (snapshot 2 in Fig. 3) and lead to an homogeneous solid solution forming at large $\overline{|\psi|}$ values (snapshot 3 in Fig. 3). Phase domains can co-exist (graphs 4,5 and 6 in Fig. 3) as expected from the $2 \mathrm{D}$ theoretical phase diagram (full line in Fig. 3). Snapshots of 2D simulations prove that the ground states in the pattern domain (hatched area in Fig. 1) are not unique and cannot be modeled by a simple hyperbolic tangent profile leading the previous computations of the phase limits between the (SS) and the (SD) domains questionable ${ }^{14}$. Extension of this analysis to three dimensions is straightforward.
Besides identification of different micro-structures, ground states of $\mathcal{L}_{S H}[\psi]$ also provides the solubility limits of precipitates in the pattern domain $\left(A_{S}\right.$ and $A_{h}$ in $2 \mathrm{D})$. These solubility limits are functions of $\bar{\psi}$. Such a dependence was systematically omitted in previous calculations of radiation-induced solubility limits ${ }^{14,28}$. Fig. 4 displays variation of these solubility limits as a function of $\Delta$ (full red lines). The comparison between theoreti-
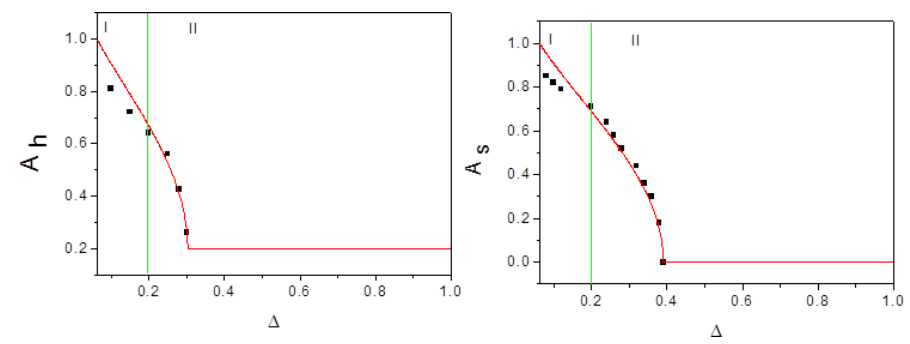

FIG. 4. Comparison of solubility limits $A_{s}$ and $A_{h}$ (full red line) calculated from the minimization of the $2 \mathrm{D} \mathrm{SH}$ functional and extracted from 2D numerical simulations (black squares) for the honeycomb (left, $\bar{\psi}=0.25$ ) and the labyrinthine (right, $\bar{\psi}=0$ ) micro-structures for different $\Delta$ values $(R=3)$.

cal (full line) and calculated from Eq. 3 (black squares) solubility limits are in very good agreement in the vicinity of the (SS) domain for which $\epsilon$ tends to zero (region II in Fig. 4) and the one mode approximation holds ${ }^{15}$. This agreement does not hold in the vicinity of the (SD) domain for which $\epsilon$ tends to $\infty$ (region I in Fig. 4). This limitation can be easily overcome introducing higher order mode expansions ${ }^{15}$.

Prediction and modelling of radiation-induced disordering is a long term goal in the advanced materials community. Our study proves that radiation-induced patterns in non-miscible alloys result from the existence of a Swift-Hohenberg functional $\mathcal{L}_{S H}$ and that the radiationinduced micro-structure in the pattern domain is not unique. All possible micro-structures, resulting from the minimization of the SH functional, can be drawn in a generic "pseudo phase diagram" highlighting the impact on the overall composition, up to now neglected ${ }^{12,14}$. Thus, this approach offers a novel quantitative method to design new classes of nano-materials by tuning not only the temperature and the flux of incident particles but also the overall composition of the alloy.

\section{ACKNOWLEDGMENTS}

This work was supported by the French initiative of basic research (CEA/RSTB) for the nuclear industry. GLM thanks the support of the Australian Institute of Nuclear Science and Engineering (AINSE) and also funding from the SAAFE scholarship. 
${ }^{1}$ G. Nicolis, I. Prigogine, Self organization in Nonequilibrium Systems: from dissipative structure to order through fluctuations, Wiley-NY, 1977.

${ }^{2}$ M. Seul, D. Andelman, Domain shapes and patterns : the phenomenology of modulated phases, Science 267 (1995) 476.

${ }^{3}$ M. C. Cross, P. C. Hohenberg, Pattern formation outside of equilibrium, Rev. Mod. Phys. 65 (1993) 851-1112.

${ }^{4}$ A. Barbu, G. Martin, A. Chamberod, Low-flux radiation-induced precipitation, J. Appl. Phys. 51 (1980) 126192.

${ }^{5}$ H. Bernas, J.-P. Attané, K.-H. Heinig, D. Halley, D. Ravelosona, A. Marty, P. Auric, C. Chappert, Y. Samson, Ordering intermetallic alloys by ion irradiation: A way to tailor magnetic media, Phys. Rev. Lett. 91 (2003) 077203.

${ }^{6} \mathrm{~N}$. Ghoniem, D. Walgraef, Instabilities and self organization in materials, vol I, Oxford Science publications, 2008.

${ }^{7}$ D. Simeone, J. Ribis, Luneville, Continuum approaches for modeling radiation-induced self-organization in materials: From the rate theory to the phase field approach, J. Mat. Resaerch. 33 (2018) 440-454.

${ }^{8}$ R. A. Enrique, P. Bellon, Nonequilibrium fluctuations, effective temperature, and effective interactions driven by irradiation of alloys, Phys. Rev. B 70 (2004) 224106.

${ }^{9}$ R. A. Enrique, K. Nordlund, R. S. Averback, P. Bellon, Simulations of dynamical stabilization of $\mathrm{AgCu}$ nanocomposites by ion-beam processing, J. of Appl. Phys. 93 (5) (2003) 2917.

${ }^{10}$ D. Simeone, G. Demange, L. Luneville, Disrupted coarsening in complex Cahn-Hilliard dynamics, Phys. Rev. E 88 (2013) 032116.

${ }^{11}$ G. Demange, L. Luneville, V. Pontikis, D. Simeone, Prediction of irradiation induced microstructures using a multiscale method coupling atomistic and phase field modeling: Application to the agcu model alloy, J. Appl. Phys. 121 (2017) 125108-125122.

${ }^{12}$ L. Luneville, K. Mallick, V. Pontikis, D. Simeone, Patterning in systems driven by non local external forces, Phys. Rev. E 94 (2016) 052126.

${ }^{13}$ R. A. Enrique, P. Bellon, Self organized $\mathrm{Cu}-\mathrm{Ag}$ nanocomposites synthesized by intermediate temperature ion-beam mixing, Appl. Phys. Lett. 78.

${ }^{14}$ R. A. Enrique, P. Bellon, Compositional patterning in systems driven by competing dynamics of different length scale, Phys. Rev. Lett. 84 (13) (2000) 2885-8.

${ }^{15} \mathrm{M}$. Cross, H. Greenside, Pattern formation and Dynamics in Non equilibrium systems, Cambridge, 2009.

${ }^{16}$ Y. Adda, M. Beyeler, G. Brebec, Monte carlo simulation of phase separation in chemically reactive binary mixture, Thin Solid Films 25 (S1) (1975) S28.

${ }^{17}$ P. Subramanian, J. Perepezko, "the ag-cu system : A phase diagram evaluation", Journal of Phase Equilibrium 14 (1993) 62

${ }^{18}$ A. G. Khatchaturyan, Theory of structural transformation in solids, Wiley Interscience, 1983.

${ }^{19}$ P. Tolédano, V. Dmitriev, Reconstructive phase transitions: in crystals and quasicrystals, World Scientific, 1996.

${ }^{20}$ A. Gras-Marti, P. Sigmund, Distortion of depth profiles during ion bombardment ii. mixing mechanisms, Nucl. Inst. and Methods B 180 (1) (1981) $211-219$.

${ }^{21}$ D. Simeone, L. Luneville, Concentration profile distortion under ion beam mixing: An example of levy flight, Phys. Rev. E 81 (2010) 021115.

${ }^{22}$ A. J. Bray, Theory of phase-ordering kinetics, Advances in Physics 43 (1994) 357-459.

${ }^{23}$ A. J. Bray, C. L. Emmott, Lifshitz-slyozov scaling for late-stage coarsening with an order-parameter-dependent mobility, Physical Review B 52 (2) (1995) R685.

${ }^{24}$ R. Enrique, P. Bellon, No equilibrium fluctuations, effective temperature and effective interactions driven by irradiation of alloys, Phys. Rev. B 70 (2004) 224106.

${ }^{25}$ K. Elder, M. Katakowski, M. Haataja, M. Grant, Modelling elasticity in crystal growth, Phys. Rev. Lett. 88 (2002) 245701.

${ }^{26} \mathrm{~K}$. Elder, M. Grant, Modelling elastic and plastic deformations in nonequilibrium processing using pfc, Phys. Rev. E 70 (2004) 051605 .

${ }^{27}$ U. Thiele, M. Archer, M. Robbins, Localized states in the conserved swift-hohenberg equation with cubic nonlinearity, Phys. Rev. E 87 (2013) 042915-1-042915-19.

${ }^{28}$ G. Martin, Phase stability under irradiation: Ballistic effects, Phys. Rev. B 30 (3) (1984) 53. 